\title{
Sleep quality in students: Associations with psychological and lifestyle factors
}

\author{
Liv C. Henrich ${ }^{1,2} \cdot$ Niki Antypa $^{1}$ • Julia F. Van den Berg ${ }^{1,3}$ (D)
}

Accepted: 26 April 2021 / Published online: 6 May 2021

(C) The Author(s) 2021

\begin{abstract}
Disturbed sleep is prevalent in adult populations and has been associated with negative health outcomes. This cross-sectional study assessed how psychological and lifestyle factors relate to subjective sleep quality in university students. In a large $(N=$ 1114) sample of Dutch university students we assessed chronotype (Morningness-Eveningness Questionnaire), depressive symptoms (Quick Inventory of Depressive Symptoms), cognitive emotion regulation styles (Cognitive Emotion Regulation Questionnaire), and substance use (caffeine, alcohol, smoking, cannabis), and analysed whether these factors were related to subjective sleep quality (Pittsburgh Sleep Quality Index). The sample was predominantly $(77.8 \%)$ female, mean age $21.1(\mathrm{SD}=$ $2.9)$ years, and $42.8 \%$ had clinically significant poor subjective sleep quality. More eveningness $(\mathrm{B}=-.05, p=.001)$, more depressive symptoms $(\mathrm{B}=.31, p=.001)$, less use of the emotion regulation strategy 'putting into perspective' $(\mathrm{B}=-.06$, $p=.02)$ and smoking $(\mathrm{B}=.53, p=.02)$ were independently associated with poorer subjective sleep quality. More eveningness, more depressive symptoms, less use of the emotion regulation style 'putting into perspective' and smoking were independently associated with poorer subjective sleep quality in a large sample of Dutch university students.
\end{abstract}

Keywords Sleep quality $\cdot$ Chronotype $\cdot$ Depressive symptoms $\cdot$ Lifestyle

We spend about one third of our lives asleep (Siegel, 2005). Sufficient and restorative sleep is considered a cornerstone of both mental and physical health (Siegel, 2005). Yet, sleep problems such as difficulty falling and remaining asleep are highly prevalent in adult populations (Buysse et al., 1989; Ohayon, 2002; Léger et al., 2000).

Research has consistently shown that disturbed sleep can have detrimental effects on physical health, psycho-social well-being and psychological functioning (Roth, 2007). For example, disturbed sleep has been associated with heart disease (Kanagala et al., 2003) and an increased risk of

Julia F. Van den Berg

j.f.van.den.berg@fsw.leidenuniv.nl

Liv C. Henrich

liv.henrich@uni-marburg.de

1 Department of Clinical Psychology, Faculty of Social Sciences, Leiden University, Wassenaarseweg 52, 2333, AK Leiden, The Netherlands

2 Present address: AG Klinische Psychologie und Psychotherapie, Philipps-Universität Marburg, Gutenbergstraße 18, D-35032 Marburg, Germany

3 Parnassia Psychiatric Institute, The Hague, The Netherlands developing neurodegenerative disorders such as Alzheimer's disease (Burke et al., 2018). Disturbed sleep can also negatively affect cognitive functioning (Diekelmann, 2014), as well as academic performance (Curcio et al., 2006) and quality of life more generally (Léger et al., 2001; Ohayon et al., 1997).

Good sleep quality, on the other hand, has positive effects on daily emotional functioning and reduced stress reactivity (Blaxton et al., 2017). Thus, it is important to determine potential modifiable risk factors of poor sleep quality so that negative health outcomes may be prevented.

Particularly young adults and students seem to be at risk of developing sleep problems (Buboltz Jr et al., 2009). Longitudinal research has shown that the transition from adolescence to adulthood is marked by several social and biological changes that may affect sleep quality. Young adults typically do not continue to execute the sleep habits acquired during childhood or adolescence (Park et al., 2019). The new lifestyle challenges young adults are faced with can lead to the development of sleep difficulties in this group. Interventions aimed at increasing sleep quality in this group would therefore need to consider these particular challenges.

The chronotype is an important factor to take into account when assessing behavioral predictors of sleep quality (Burke 
et al., 2015). Chronotype refers to a person's preferred activity and sleep schedules and are governed by internal circadian rhythm timings (Kerkhof, 1985). A preference for late activity and late sleep onset is described as an evening chronotype. Throughout the lifespan circadian timings change from an early preference in childhood to a late preference during adolescence, peaking around the age of 20 . Hereafter, another transition toward the earlier chronotype occurs, which has been described as a marker for the end of adolescence (Roenneberg et al., 2004). The evening chronotype has been associated with poorer sleep quality (Vitale et al., 2015). Furthermore, evening types have an increased risk of experiencing depressive symptoms (Kivelä et al., 2018), and this relationship was mediated by sleep quality in a student sample (Van den Berg et al., 2018).

In terms of psychological health, young adulthood in particular represents a vulnerable time for the onset of psychiatric disorders (Kessler et al., 2005). Research suggests complex bidirectional relations between poor sleep and mood disorders (Ohayon \& Roth, 2003). Sleep disturbances can be a clinically valuable predictive sign in depression, as they tend to precede the onset of a depressive episode or relapse (Alvaro et al., 2013; Baglioni et al., 2011; Ford \& Kamerow, 1989; Ohayon \& Roth, 2003). However, some research also suggests a reverse relationship in that depressive symptoms predict worse sleep quality (Kennedy et al., 1991). In a student sample, poor sleep quality was associated with higher selfreported negative mood, and anxiety and stress were the strongest predictors of subjective sleep quality (Lund et al., 2010). This line of work indicates that mood and cognitive reactions to stress are likely to play an important role in the complex psychological interactions that affect sleep quality. Young adulthood is a period in which cognitive regulation skills develop. Imaging studies comparing children, adolescents and young adults show linear relationships between age and the activation of prefrontal cortex regions required for complex emotion regulation involving cognitive control (McRae et al., 2012). One recent development in sleep research is to assess the relationships between cognitive emotion regulation skills, psychological health and sleep parameters (Watts \& Norbury, 2017).

An important modifiable lifestyle risk factor for students is the increased access to and use of stimulants (e.g., caffeine) and sedatives (e.g., alcohol, cannabis), which may influence sleep parameters (Lund et al., 2010). In particular, caffeine is the most commonly used psychoactive substance worldwide (Clark \& Landolt, 2017). A recent systematic review of epidemiologic studies and clinical trials concluded that caffeine intake negatively impacts subjective and objective sleep quality (Clark \& Landolt, 2017). Specifically, evening caffeine intake equivalent of a double espresso was shown to induce a circadian phase delay of $40 \mathrm{~min}$ (Burke et al., 2015).
Only few studies have comprehensively assessed the behavioral, psychological and cognitive predictors of sleep quality in students. This is important as young adulthood is a sensitive time for experiencing poor sleep quality (Lund et al., 2010), and is characterized by several developmental transitions: changes in chronotype, increased vulnerability for affective disorders, biological developments in cognitive regulation capabilities as well as more access to substances. We aim to examine how the combination of these factors affects subjective sleep quality in this phase. Individually some of these variables have been studied previously, however, not in a combined model, which we expect to represent the transition from adolescence to adulthood more accurately. Further novel elements of this study are that we considered cognitive emotion regulation as a potential risk factor, as well as nonproblematic levels of substance use. To our knowledge no other studies have assessed the combination of these factors before. Specifically, we hypothesized that a later chronotype, more depressive symptoms, increased use of maladaptive cognitive emotion regulation strategies, and more substance use (caffeine, alcohol, smoking, cannabis) would be associated with lower subjective sleep quality.

\section{Method}

\section{Participants and Procedure}

A total of 1114 Dutch-speaking University students (77.8\% female; $22.2 \%$ male) from Leiden, The Hague, Delft, Rotterdam and Amsterdam participated in this crosssectional study. Inclusion criteria were: being enrolled as a student (full-time or part-time), being $\geq 18$ years of age and being fluent in Dutch. Participants were between 17 and 56 years old, with an average age of $21.1( \pm 2.9)$ years. Data were collected in March of 2016, 2017 and 2018. Each year data collection ended as daylight saving time began, so that difficulties with adjusting to daylight saving time would not influence the data. Students were recruited with flyers distributed throughout University buildings and through the social networks of the students who collected the data. Participants were provided a link through which they could read information about the study, sign an online informed consent form and complete questionnaires. Participation was rewarded with a lottery to win small monetary amounts $\leq € 50$. The study protocol and informed consent were approved by the Institutional Review Board, and all subjects gave informed consent.

\section{Measures}

The socio-demographic variables age, gender and education level were recorded. 
Sleep Quality Subjective sleep quality was assessed with a Dutch translation of the Pittsburgh Sleep Quality Index (PSQI; Buysse et al., 1989). The PSQI is a 19-item questionnaire assessing seven components of sleep quality (subjective sleep quality; sleep latency; sleep duration; habitual sleep efficiency; sleep disturbances; sleep medication use; and daytime functioning) over the last month. The combined scores range from 0 (no difficulty) to 21 (severe difficulties) and higher scores indicate worse sleep quality. A total PSQI score of $>5$ indicates clinically significant sleep quality problems (Buysse et al., 1989). Based on the seven PSQI components it has an internal consistency score of Cronbach's $\mathrm{a}=0.66$.

Chronotype We assessed chronotype using the Morningness/ Eveningness Questionnaire (MEQ) (Horne \& Östberg, 1976). It is a widely used instrument consisting of 19 items assessing preferred daily activity times and preferred sleep and wake times. Total scores range from 18 to 86 , where low scores $(<42)$ indicate eveningness and higher scores $(>58)$ indicate morningness. The MEQ has a high internal consistency in our sample with Cronbach's a $=0.87$.

Depressive Symptoms The Quick Inventory of Depressive Symptomatology- Self-Rated (QIDS-SR; Rush et al., 2003) was used to assess depressive symptoms. This 16-item selfreport instrument measures the severity and frequency of depressive symptoms based on the nine DSM-IV depression domains (sad mood; concentration; self-criticism; suicidal ideation; interest; energy/fatigue; sleep disturbances; changes in appetite/weight; psychomotor agitation/retardation) over the last week. Total scores range between 0 (no depressive symptoms) to 27 (severe depressive symptoms). In our sample the QIDS-SR has a Cronbach's a $=0.78$.

Cognitive Emotion Regulation Emotion regulation was assessed using the Cognitive Emotion Regulation Questionnaire (CERQ; Garnefski \& Kraaij, 2007). It consists of 36 questions measuring nine subscales, namely 'selfblame', 'acceptance', 'rumination', 'positive refocusing', 'refocus on planning', 'positive reappraisal', 'putting into perspective', 'catastrophizing', and 'blaming others'. Respondents indicate on a 5-point Likert scale ranging from 1 (almost never) to 5 (almost always) how often they use each cognitive style in response to a negative event. Scores for each subscale range between 4 and 20 and higher scores indicate more frequent use of the corresponding emotion regulation strategy. In our sample, the CERQ has high internal consistency with Cronbach's a levels ranging between 0.76 and 0.83 for all subscales.

Substance Use We assessed average weekly alcohol and caffeine consumption, smoking cigarettes and cannabis use over the past month with a 7-item questionnaire. The amount of caffeine intake (in $\mathrm{mg}$ ) was calculated based on the average number of caffeinated beverages (coffee, tea, and caffeinated soft drinks) per week, using guidelines from the Dutch Nutrition Centre (Voedingscentrum, 2019). Alcohol use was measured as average number of alcoholic beverages per week. Smoking and cannabis use were dichotomized (yes/no).

\section{Data Analyses}

To assess the possible predictors of subjective sleep quality (PSQI total score) we performed a multiple regression analysis where sum scores of the continuous variables (MEQ, QIDS-SR, all CERQ subscales, caffeine consumption, alcohol consumption), the categorical variables (smoking, cannabis use), and gender and age were entered in one step. The sleep disturbance component (4 items) in the QIDS-SR was excluded from the sum score because it represents a component of the PSQI outcome measure and might artificially inflate the regression coefficient for depressive symptoms.

Regression Model Validation In order to produce a more robust analysis, we used the bootstrap method on the whole sample to calculate the regression coefficients (significance level at $p=.05$ ). Additionally, to assess overfitting, we employed a validation procedure on a hold-out sample. We estimated the regression model on a training sample (random selection of $80 \%$ of the sample) and then tested the model in the hold-out sample (remaining $20 \%$ of the sample). Based on the model estimated on the training sample, predicted scores (on the outcome variable) were calculated for the hold-out sample and compared to the observed values. The model was considered to be robust (good predictive value), if the standard errors of the estimate remained constant in the training sample and the hold-out sample.

Missing Data Initially 1393 participants started filling in the questionnaire. We removed cases who were not students $(N=55)$ and cases who only filled in the demographic data or only filled in the first questionnaire (MEQ) in the set $(N=73)$ and those who missed all components of the outcome variable PSQI $(N=35)$. There were also participants $(N=116)$ who did not fill in any items of the last questionnaire in the set (CERQ), who were removed. This rendered the final analysis data set of $N=1114$ with no missing values. For participants who missed only one item in the sleep efficiency component (bed times, get up times, habitual sleep duration) of the PSQI $(N=4)$, we assumed the best score for this component (a sleep efficiency of $>85 \%$ ). Similarly we assumed the best item score $(0)$ for participants who missed one of the two day-time functioning component items of the PSQI $(N=3)$. 


\section{Results}

\section{Descriptive Statistics}

Table 1 summarizes the descriptive statistics of all variables. The mean PSQI score for the sample was $M=5.6( \pm 2.8)$, indicating clinically relevant poor sleep quality, and $42.8 \%$ of participants scored above this threshold.

We conducted a multiple linear regression analysis with bootstrap (requesting bias corrected confidence intervals) entering all variables in one step to predict the total PSQI score (Table 2). Among the predictors there were no correlations that exceeded a value of .7, indicating that they are not multicollinear. A complete correlation matrix can be found as online supplementary material. The model was significant, $F(17,1114)=25.77, p=.001$. The amount of explained variance was $28.6 \%\left(R^{2}=0.286\right)$, which indicates a large effect size (Cohen's $\left.f^{2}=0.4\right)$. More depressive symptoms (higher scores on QIDS-SR) were associated with poorer subjective sleep quality (higher PSQI scores), $\mathrm{B}=.31, p=.001,95 \%$ $\mathrm{CI}=.27, .36$. An earlier chronotype was associated with better sleep quality, $\mathrm{B}=-.05, p=.001,95 \% \mathrm{CI}=-.06,-.03$. Higher scores on the 'putting into perspective' CERQ subscale were associated with better subjective sleep quality, $\mathrm{B}=-.06$, $p=.02,95 \% \mathrm{CI}=-.11,-.01$. Smoking cigarettes was

Table 1 Descriptive statistics for all variables $(N=1114)$

\begin{tabular}{lll}
\hline & N $(\%)$ & M $( \pm$ SD $)$ \\
\hline Age & -- & $21.1( \pm 2.9)$ \\
Gender (female) & $867(77.8)$ & - \\
Subjective sleep quality (PSQI total) & -- & $5.6( \pm 2.8)$ \\
Chronotype (MEQ) & - & $46.9( \pm 9.8)$ \\
Depressive Symptoms (QIDS-SR) & - & $4.8( \pm 4.2)$ \\
Rumination (CERQ) & - & $11.2( \pm 3.8)$ \\
Catastrophizing (CERQ) & - & $6.4( \pm 2.7)$ \\
Self-blame (CERQ) & - & $9.8( \pm 3.3)$ \\
Other-blame (CERQ) & - & $6.3( \pm 2.4)$ \\
Positive refocusing (CERQ) & - & $10.7( \pm 3.5)$ \\
Refocus on planning (CERQ) & - & $13.2( \pm 3.4)$ \\
Positive reappraisal (CERQ) & - & $12.7( \pm 3.6)$ \\
Putting into perspective (CERQ) & - & $12.1( \pm 3.7)$ \\
Acceptance (CERQ) & - & $12.1( \pm 3.3)$ \\
Caffeine (mg/week) & - & $551( \pm 705)$ \\
Alcohol (beverages/week) & - & $4.9( \pm 7.0)$ \\
Smoking cigarettes (yes) & $228(20.5)$ & - \\
Cannabis use (yes) & $66(5.9)$ & - \\
\hline
\end{tabular}

Notes. $\mathrm{N}=$ sample; $\%$ = percentage $\mathrm{M}=$ mean; $\mathrm{SD}=$ standard deviation; PSQI = Pittsburgh Sleep Quality Index; MEQ = Morningness/ Eveningness Questionnaire; QIDS-SR = Quick Inventory of Depressive Symptomatology - Self Rated; CERQ = Cognitive Emotion Regulation Questionnaire; $\mathrm{mg}=$ milligram associated with poorer subjective sleep quality, $\mathrm{B}=.53$, $p=.02,95 \% \mathrm{CI}=.10, .98$.

Validation A multiple regression was run on a random $80 \%$ training sample $(N=881)$. The model was significant, $F(17$, $863)=19.78, p=.001$. The amount of explained variance for the model was also $28.0 \%\left(R^{2}=0.28\right)$ rendering a large effect size (Cohen's $f^{2}=0.39$ ). Similar to the bootstrapped coefficients in whole sample, the predictors that contributed to the model in the training sample were: MEQ $(\mathrm{B}=-.05, p=.001$, $95 \% \mathrm{CI}=-.07,-.03)$, $\mathrm{QIDS}(\mathrm{B}=.31, p=.001,95 \% \mathrm{CI}=.27$, $.36)$, and smoking cigarettes $(\mathrm{B}=.76, p=.001,95 \% \mathrm{CI}=.32$, 1.20). Notably, also alcohol consumption $(\mathrm{B}=-.03, p=.03$, $95 \% \mathrm{CI}=-.06,-.003)$ was a significant predictor of subjective sleep quality, whereas 'putting into perspective' was not ( $\mathrm{B}=-.03, p=.24,95 \% \mathrm{CI}=-.09, .02)$. This model was then used to calculate predicted PSQI scores for the hold-out sample $(N=233)$. The correlation between predicted and observed values in the training sample was $r=.530$, while in the holdout sample it was $r=.531$, indicating that the model predicts PSQI scores almost equally well in the training and the test sample. We further calculated the standard error of the estimate for the hold-out sample $(S E=2.3)$ to compare it to the one obtained from the training sample $(S E=2.4)$. Since both values are close to each other we conclude that the results are stable in an unselected group.

\section{Discussion}

This large cross-sectional study aimed to assess how several important lifestyle and psychological factors might contribute to deteriorating sleep quality in young adulthood. Our analysis showed that chronotype (more eveningness), depressive symptoms, less use of the cognitive emotion regulation strategy 'putting into perspective', and smoking were significant predictors of poor subjective sleep quality, controlling for age and gender. Together these variables explained $29 \%$ of the variance in subjective sleep quality. Caffeine consumption, cannabis use and the other eight cognitive emotion regulation styles were not significantly associated with subjective sleep quality. In the validation procedure alcohol consumption was also significantly associated with better subjective sleep quality, whereas 'putting into perspective' was not.

\section{Chronotype}

A later chronotype was the second largest predictor of subjective sleep quality. Our finding is in line with existing literature on chronotypes and sleep quality. Nearly $28 \%$ of the sample were evening types; given that the peak in eveningness across the lifespan occurs around the age of 20 (Roenneberg et al., 2004), and the average age in our sample was 21 , our sample 
Table 2 Regression 1 - Bootstrapped coefficients with PSQI total score as outcome

\begin{tabular}{|c|c|c|c|c|c|c|}
\hline \multirow[t]{3}{*}{ Mode } & & \multirow[t]{3}{*}{$\mathrm{B}$} & \multicolumn{4}{|l|}{ Bootstrap } \\
\hline & & & \multirow[t]{2}{*}{ Std. Error } & \multirow[t]{2}{*}{$p$ value (2-tailed) } & \multicolumn{2}{|c|}{ 95\% Confidence Interval } \\
\hline & & & & & Lower & Upper \\
\hline \multirow[t]{18}{*}{1} & (Constant) & 5.27 & .88 & .00 & 3.27 & 6.91 \\
\hline & Gender & .29 & .18 & .10 & -.05 & .63 \\
\hline & Age & .02 & .03 & .41 & -.02 & .08 \\
\hline & Chronotype (MEQ) & -.05 & .01 & $<.001$ & -.06 & -.03 \\
\hline & Depressive symptoms (QIDS-SR) & .31 & .02 & $<.001$ & .27 & .36 \\
\hline & Rumination (CERQ) & -.02 & .02 & .44 & -.06 & .02 \\
\hline & Catastrophizing (CERQ) & .03 & .04 & .48 & -.05 & .09 \\
\hline & Self-blame (CERQ) & .02 & .03 & .43 & -.03 & .08 \\
\hline & Other-blame (CERQ) & -.01 & .04 & .83 & -.08 & .07 \\
\hline & Positive refocusing (CERQ) & .01 & .03 & .61 & -.04 & .07 \\
\hline & Refocus on planning (CERQ) & -.02 & .03 & .55 & -.09 & .04 \\
\hline & Positive reappraisal (CERQ) & .04 & .03 & .28 & -.03 & .11 \\
\hline & Putting into perspective (CERQ) & -.06 & .03 & .02 & -.11 & -.01 \\
\hline & Acceptance (CERQ) & .02 & .03 & .41 & -.03 & .08 \\
\hline & Caffeine (mg/week) & $5.21 \mathrm{E}-5$ & .00 & .66 & .00 & .00 \\
\hline & Alcohol (beverages/week) & -.02 & .01 & .10 & -.04 & .01 \\
\hline & Cannabis use (yes) & -.05 & .42 & .92 & -.85 & .87 \\
\hline & Smoking cigarettes (yes) & .53 & .22 & .02 & .10 & .98 \\
\hline
\end{tabular}

Notes. Bootstrap based on 500 bootstrap samples; MEQ = Morningness/Eveningness Questionnaire; QIDS-SR = Quick Inventory of Depressive Symptomatology - Self Rated; CERQ = Cognitive Emotion Regulation Questionnaire; $\mathrm{mg}=$ milligram

represents a fair estimate of evening types in this population. We suspect that a large proportion of the sample is yet to transition toward an earlier chronotype and is currently more vulnerable to sleep quality problems. Evening types were shown to have reduced sleep quality during the week as compared to morning and intermediate types (Vitale et al., 2015; Wittmann et al., 2006). This may be explained by evening types having to adhere to social and professional schedules that are misaligned with their chronotype and only being able to achieve comparable levels of sleep quality as earlier types during the weekend (Vitale et al., 2015). It is possible that the same explanation holds for our student sample, in that evening types also adhere to their study and social schedule during the week, which may be misaligned to their circadian rhythms.

\section{Depressive Symptoms}

The strongest predictor of poorer subjective sleep quality (adjusted for the other variables) was depressive symptoms. Our finding supports existing research on the relationship between depression and poor sleep quality (Kennedy et al., 1991; Ohayon \& Roth, 2003). Other epidemiological studies conclude that somatic or emotional distress presents the best predictor of insomnia in adults (Ford \& Kamerow, 1989). In a sample closer to our own, a large study of college students found that emotional distress was the largest predictor of poor sleep quality (Lund et al., 2010). In open-ended questions, a large proportion of students in this study named thought patterns typical for depressive symptoms (such as worries about the future and school) as the reason for their difficulties with sleep (Lund et al., 2010).

Since our study was cross-sectional, we cannot establish temporal precedence and the term 'predictor' in this context is strictly statistical. Nevertheless, the strong association between depressive symptoms and sleep are further supported by research showing that $90 \%$ of patients with depression present with sleep disturbances (Franzen \& Buysse, 2008) and that untreated sleep problems increase the likelihood of relapse in depression (Ford \& Kamerow, 1989; Franzen \& Buysse, 2008; Ohayon \& Roth, 2003). One possible explanation is that depression and sleep parameters depend on shared regulatory neurotransmitter pathways (Wulff et al., 2010), which may support the idea that treating depressive symptoms and sleep problems should go hand in hand (Cunningham \& Shapiro, 2018).

\section{Cognitive Emotion Regulation}

Based on theoretical models of emotion regulation (Baglioni et al., 2010), we expected that disturbances in emotion regulation typically seen in the context of heightened cognitive 
arousal, such as rumination and worry, would be related to sleep quality. Particularly, rumination has been shown to increase pre-sleep arousal and predict reduced sleep quality in young adults with depressive symptoms (Pillai et al., 2014) and healthy students (Takano et al., 2012). Longitudinal research in students further showed that problems with emotion regulation relate bi-directionally to sleep problems (Tavernier \& Willoughby, 2015).

Eight of nine emotion regulation styles assessed in this study did not predict sleep quality significantly. Only less use of 'putting into perspective' was associated with poorer sleep quality. However, this variable was not significant in the validation procedure. A potential explanation could be that in order to observe the effects of daily emotion regulation, sleep quality needs to be assessed the same night. Perhaps momentary measurements that are sensitive to fluctuations in stress-responsiveness, cognitive arousal and same-night sleep quality could provide a useful approach to studying cognitive-emotional predictors of sleep quality in the future (see: Gilbert et al., 2017 for an example in diurnal cortisol functioning). Another distinction that we did not make and could help explain our finding is that effective emotion regulation may depend on coping style flexibility. For instance one emotion regulation style may be adaptive in response to a particular stressor, but not in response to another (Kahn et al., 2013). Another possible explanation for the results may be that emotion regulation plays a more important role for a sub-group of the sample, such as those individuals with elevated symptoms of depression or those who have already developed the cognitive capabilities to use complex emotion regulation strategies (McRae et al., 2012). Because we controlled for depressive symptoms in the regression analysis, emotion regulation styles may not have emerged as independent predictors of sleep quality.

\section{Substance Use}

In our study, caffeine was not a significant predictor of sleep quality. This is somewhat surprising as caffeine is a wellstudied substance known to affect wakefulness and sleep (Clark \& Landolt, 2017). In our sample caffeine consumption was low in general, which may help to explain this result. Another explanation could be that people moderate their caffeine intake according to their own levels of caffeine sensitivity (Rétey et al., 2007).

Alcohol was not a significant predictor in our main analysis. Yet, it was a significant predictor of better sleep quality in our validation procedure. Although this result requires replication before firm conclusions can be drawn, the finding is in accordance with other mixed results about the effects of alcohol in different dosages on health and sleep. A review on the effects of alcohol on objective sleep parameters in healthy subjects concluded that, at all dosages, alcohol reduced time to sleep onset and seemed to produce a more consolidated (less waking after sleep onset) first half of sleep. This, however, was usually followed by increased interruptions in the second half (Ebrahim et al., 2013).

In line with the literature on smoking, it was a significant predictor of poorer sleep quality in our main analysis. This finding is supported by research showing that smokers tend to report more sleep-related psychosomatic symptoms, feel less rested after sleep (Wittmann et al., 2010), have more difficulties initiating and maintaining sleep, and show more daytime sleepiness (Wetter \& Young, 1994). Nicotine acts on several (nicotinergic acetylcholine, glutaminergic, dopaminergic and serotinergic) neurotransmitter pathways in the brain and thereby affects states of wakefulness and sleepiness as well as sleep architecture (Jaehne et al., 2009). Thus nicotine's effects on sleep quality, specifically its effect on delayed sleep onset latency and sleep architecture can likely be attributed to its stimulant effects on the brain, while frequent sleep disturbances may be explained by the experience of nightly withdrawal symptoms (Wittmann et al., 2010).

Cannabis use was not a significant predictor of subjective sleep quality. A recent critical review of randomized controlled trials and open label studies concluded that many studies show some evidence that cannabis use is associated with greater subjective sleep quality, shorter sleep latency, longer sleep duration and fewer episodes of wakefulness during the night (Kuhathasan et al., 2019). However, most studies lacked placebo controls and did not use objective measures of sleep quality (Kuhathasan et al., 2019). Our study did not find such associations, but only a small proportion of our sample reported consuming cannabis so a potential lack of power may also explain our result.

Strengths of the study are that we had a large sample $(N=$ 1114) and thus high overall power. In addition, the survey was anonymous which likely reduced socially desirable responses. We also sought to assess a more comprehensive model of changing lifestyle and psychological factors relevant in young adulthood than many studies previously. Additionally, we used a bootstrap and a validation method to assess how robust our findings are.

There are several limitations to point out about our study. First, even though the dataset is large, it is cross-sectional, thus we cannot assert whether the independent variables also act as predictors in a temporal sense. Second, we aimed to identify factors that are relevant and undergoing changes in college students' lives, yet our list is not exhaustive. There may be other important factors we did not consider, such as academic stress or levels of physical activity, which future studies on the topic could consider.

Third, because we used posters and flyers and the social network of the students who carried out the data collection to recruit participants, we cannot exclude the possibility of selfselection bias and reduced generalizability resulting from our recruitment strategy. Forth, since women were strongly over- 
represented in our sample, we caution readers that our results may not generalize to the wider student population. Fifth, for practical reasons we measured sleep quality with a subjective (self-report) measure, so recall bias could have occurred. Another limitation is that only self-report measures were used in this study, which might have contributed to inflated correlations among variables.

Because recent research highlights that risk factors for poor sleep quality in young adulthood likely to differ from risk factors in adolescence or adulthood (Park et al., 2019), our results may help shed some light on to the unique challenges that young adults face regarding sleep during their student life. Furthermore, our results may help guide higher education institutions and healthcare professionals working with students to increase sleep quality, academic performance (Curcio et al., 2006) and reduce distress during psychologically vulnerable times (Baglioni et al., 2011). Additionally, our results stress that students might benefit from more flexible schedules and opportunities to study at times that suit their chronobiology best (for example by making lecture materials available online). This is particularly important for evening chronotypes, who, due to insufficient sleep and social jetlag, might not be able to reach their optimal cognitive and academic performance (Wittmann et al., 2006).

By simultaneously assessing a set of developmentally relevant psychological and lifestyle factors, this large scale study found that more eveningness, more depressive symptoms, less use of the cognitive emotion regulation style 'putting into perspective', and smoking cigarettes significantly predicted lower sleep quality in students. 'Putting into perspective' and alcohol were only significant predictors in some analyses and thus these findings require replication. Further research with momentary measurements and longitudinal designs will be needed to better disentangle the complex relationships between behavior, psychopathology and sleep quality in this age group.

Supplementary Information The online version contains supplementary material available at https://doi.org/10.1007/s12144-021-01801-9.

Authors' Contribution Statements Julia van den Berg and Niki Antypa conceived and designed the study. Data was collected by groups of students under supervision of Julia van den Berg and Niki Antypa. Liv Henrich performed data analysis and wrote the first draft of the manuscript. All authors commented on previous versions of the manuscript. All authors read and approved the final manuscript.

Data, Materials and/or Code Availability The data and code can be made available upon request.

\section{Declarations}

Ethics Approval This study was performed in line with the principles of the Declaration of Helsinki. Approval was granted by the Ethics Committee of the Institute of Psychology, Leiden University (6 March 2018, number CEP18-0226/116).
Consent Informed consent was obtained from all individual participants included in the study.

Conflicts of Interest/Competing Interests The authors have no relevant financial or non-financial interests to disclose.

Open Access This article is licensed under a Creative Commons Attribution 4.0 International License, which permits use, sharing, adaptation, distribution and reproduction in any medium or format, as long as you give appropriate credit to the original author(s) and the source, provide a link to the Creative Commons licence, and indicate if changes were made. The images or other third party material in this article are included in the article's Creative Commons licence, unless indicated otherwise in a credit line to the material. If material is not included in the article's Creative Commons licence and your intended use is not permitted by statutory regulation or exceeds the permitted use, you will need to obtain permission directly from the copyright holder. To view a copy of this licence, visit http://creativecommons.org/licenses/by/4.0/.

\section{References}

Alvaro, P. K., Roberts, R. M., \& Harris, J. K. (2013). A systematic review assessing bidirectionality between sleep disturbances, anxiety, and depression. SLEEP, 36(7), 1059-1068.

Baglioni, C., Spiegelhalder, K., Lombardo, C., \& Riemann, D. (2010). Sleep and emotions: A focus on insomnia. Sleep Medicine Reviews, 14(4), 227-238.

Baglioni, C., Battagliese, G., Feige, B., Spiegelhalder, K., Nissen, C., Voderholzer, U., Lombardo, C., \& Riemann, D. (2011). Insomnia as a predictor of depression: A meta-analytic evaluation of longitudinal epidemiological studies. Journal of Affective Disorders, 135(1-3), 10-19.

Blaxton, J. M., Bergeman, C. S., Whitehead, B. R., Braun, M. E., \& Payne, J. D. (2017). Relationships among nightly sleep quality, daily stress, and daily affect. Journals of Gerontology: Psychological Sciences, 72(3), 363-372.

Buboltz Jr., W., Jenkins, S. M., Soper, B., Woller, K., Johnson, P., \& Faes, T. (2009). Sleep habits and patterns of college students: An expanded study. Journal of College Counseling, 12(2), 113-124.

Burke, T. M., Markwald, R. R., McHill, A. W., Chinoy, E. D., Snider, J. A., Bessman, S. C., et al. (2015). Effects of caffeine on the human circadian clock in vivo and in vitro. Science Translational Medicine, 7(305), 305ra146-305ra146.

Burke, S. L., Cadet, T., Alcide, A., O’Driscoll, J., \& Maramaldi, P. (2018). Psychosocial risk factors and Alzheimer's disease: the associative effect of depression, sleep disturbance, and anxiety. Aging \& mental health, 22(12), 1577-1584. https://doi.org/10.1080/ 13607863.2017.1387760.

Buysse, D. J., Reynolds III, C. F., Monk, T. H., Berman, S. R., \& Kupfer, D. J. (1989). The Pittsburgh sleep quality index: A new instrument for psychiatric practice and research. Psychiatry Research, 28(2), 193-213.

Clark, I., \& Landolt, H. P. (2017). Coffee, caffeine, and sleep: A systematic review of epidemiological studies and randomized controlled trials. Sleep Medicine Reviews, 31, 70-78.

Cunningham, J. E., \& Shapiro, C. M. (2018). Cognitive Behavioural therapy for insomnia (CBT-I) to treat depression: A systematic review. Journal of Psychosomatic Research, 106, 1-12.

Curcio, G., Ferrara, M., \& De Gennaro, L. (2006). Sleep loss, learning capacity and academic performance. Sleep Medicine Reviews, 10(5), 323-337.

Diekelmann, S. (2014). Sleep for cognitive enhancement. Frontiers in Systems Neuroscience, 8, 46.

Ebrahim, I. O., Shapiro, C. M., Williams, A. J., \& Fenwick, P. B. (2013). Alcohol and sleep I: Effects on normal sleep. Alcoholism: Clinical and Experimental Research, 37(4), 539-549. 
Ford, D. E., \& Kamerow, D. B. (1989). Epidemiologic study of sleep disturbances and psychiatric disorders: An opportunity for prevention? Journal of the American Medical Association, 262(11), $1479-1484$.

Franzen, P. L., \& Buysse, D. J. (2008). Sleep disturbances and depression: Risk relationships for subsequent depression and therapeutic implications. Dialogues in Clinical Neuroscience, 10(4), 473-481.

Garnefski, N., \& Kraaij, V. (2007). The cognitive emotion regulation questionnaire. European Journal of Psychological Assessment, 23(3), 141-149.

Gilbert, K., Mineka, S., Zinbarg, R. E., Craske, M. G., \& Adam, E. K. (2017). Emotion regulation regulates more than emotion: Associations of momentary emotion regulation with diurnal cortisol in current and past depression and anxiety. Clinical Psychological Science, 5(1), 37-51.

Horne, J. A., \& Östberg, O. (1976). A self-assessment questionnaire to determine morningness-eveningness in human circadian rhythms. International Journal of Chronobiology, 4(2), 97-110.

Jaehne, A., Loessl, B., Bárkai, Z., Riemann, D., \& Hornyak, M. (2009). Effects of nicotine on sleep during consumption, withdrawal and replacement therapy. Sleep Medicine Reviews, 13(5), 363-377.

Kahn, M., Sheppes, G., \& Sadeh, A. (2013). Sleep and emotions: Bidirectional links and underlying mechanisms. International Journal of Psychophysiology, 89(2), 218-228.

Kanagala, R., Murali, N. S., Friedman, P. A., Ammash, N. M., Gersh, B. J., Ballman, K. V., M. Shamsuzzaman, A. S., \& Somers, V. K. (2003). Obstructive sleep apnea and the recurrence of atrial fibrillation. Circulation, 107(20), 2589-2594.

Kennedy, G. J., Kelman, H. R., \& Thomas, C. (1991). Persistence and remission of depressive symptoms in late life. The American Journal of Psychiatry, 148(2), 174-178.

Kerkhof, G. A. (1985). Inter-individual differences in the human circadian system: A review. Biological Psychology, 20(2), 83-112.

Kessler, R. C., Berglund, P., Demler, O., Jin, R., Merikangas, K. R., \& Walters, E. E. (2005). Lifetime prevalence and age-of-onset distributions of DSM-IV disorders in the National Comorbidity Survey Replication. Archives of General Psychiatry, 62(6), 593-602.

Kivelä, L., Papadopoulos, M. R., \& Antypa, N. (2018). Chronotype and psychiatric disorders. Current sleep medicine reports, 4(2), 94-103.

Kuhathasan, N., Dufort, A., MacKillop, J., Gottschalk, R., Minuzzi, L., \& Frey, B. N. (2019). The use of cannabinoids for sleep: A critical review on clinical trials. Experimental and Clinical Psychopharmacology, 27(4), 383-401.

Léger, D., Guilleminault, C., Dreyfus, J. P., Delahaye, C., \& Paillard, M. (2000). Prevalence of insomnia in a survey of 12778 adults in France. Journal of Sleep Research, 9(1), 35-42.

Léger, D., Scheuermaier, K., Philip, P., Paillard, M., \& Guilleminault, C. (2001). SF-36: Evaluation of quality of life in severe and mild insomniacs compared with good sleepers. Psychosomatic Medicine, 63(1), 49-55.

Lund, H. G., Reider, B. D., Whiting, A. B., \& Prichard, J. R. (2010). Sleep patterns and predictors of disturbed sleep in a large population of college students. Journal of Adolescent Health, 46(2), 124-132.

McRae, K., Gross, J. J., Weber, J., Robertson, E. R., Sokol-Hessner, P., Ray, R. D., Gabrieli, J. D. E., \& Ochsner, K. N. (2012). The development of emotion regulation: An fMRI study of cognitive reappraisal in children, adolescents and young adults. Social Cognitive and Affective Neuroscience, 7(1), 11-22.

Ohayon, M. M. (2002). Epidemiology of insomnia: What we know and what we still need to learn. Sleep Medicine Reviews, 6(2), 97-111.

Ohayon, M. M., \& Roth, T. (2003). Place of chronic insomnia in the course of depressive and anxiety disorders. Journal of Psychiatric Research, 37(1), 9-15.
Ohayon, M. M., Caulet, M., \& Guilleminault, C. (1997). How a general population perceives its sleep and how this relates to the complaint of insomnia. SLEEP, 20(9), 715-723.

Park, H., Chiang, J. J., Irwin, M. R., Bower, J. E., McCreath, H., \& Fuligni, A. J. (2019). Developmental trends in sleep during adolescents' transition to young adulthood. Sleep Medicine, 60, 202-210.

Pillai, V., Steenburg, L. A., Ciesla, J. A., Roth, T., \& Drake, C. L. (2014). A seven day actigraphy-based study of rumination and sleep disturbance among young adults with depressive symptoms. Journal of Psychosomatic Research, 77(1), 70-75.

Rétey, J. V., Adam, M., Khatami, R., Luhmann, U. F. O., Jung, H. H., Berger, W., \& Landolt, H. P. (2007). A genetic variation in the adenosine A2A receptor gene (ADORA2A) contributes to individual sensitivity to caffeine effects on sleep. Clinical Pharmacology \& Therapeutics, 81(5), 692-698.

Roenneberg, T., Kuehnle, T., Pramstaller, P. P., Ricken, J., Havel, M., Guth, A., \& Merrow, M. (2004). A marker for the end of adolescence. Current Biology, 14(24), R1038-R1039.

Roth, T. (2007). Insomnia: Definition, prevalence, etiology, and consequences. Journal of Clinical Sleep Medicine, 3(5 Suppl), S7.

Rush, A. J., Trivedi, M. H., Ibrahim, H. M., Carmody, T. J., Arnow, B., Klein, D. N., et al. (2003). The 16-item quick inventory of depressive symptomatology (QIDS), clinician rating (QIDS-C), and selfreport (QIDS-SR): A psychometric evaluation in patients with chronic major depression. Biological Psychiatry, 54(5), 573-583.

Siegel, J. M. (2005). Clues to the functions of mammalian sleep. Nature, 437(7063), 1264-1271.

Takano, K., Iijima, Y., \& Tanno, Y. (2012). Repetitive thought and selfreported sleep disturbance. Behavior Therapy, 43(4), 779-789.

Tavernier, R., \& Willoughby, T. (2015). A longitudinal examination of the bidirectional association between sleep problems and social ties at university: The mediating role of emotion regulation. Journal of Youth and Adolescence, 44(2), 317-330.

Van den Berg J. F., Kivelä L., \& Antypa N. (2018). Chronotype and depressive symptoms in students: An investigation of possible mechanisms. Chronobiology International, 35(9), 1248-1261. https://doi.org/10.1080/07420528.2018.1470531.

Vitale, J. A., Roveda, E., Montaruli, A., Galasso, L., Weydahl, A., Caumo, A., \& Carandente, F. (2015). Chronotype influences activity circadian rhythm and sleep: Differences in sleep quality between weekdays and weekend. Chronobiology International, $32(3), 405-415$.

Voedingscentrum. Caffeine information (2019) . Retrieved from (last accessed 18.09.2019): https://www.voedingscentrum.nl/ encyclopedie/cafeine

Watts, A. L., \& Norbury, R. (2017). Reduced effective emotion regulation in night owls. Journal of Biological Rhythms, 32(4), 369-375.

Wetter, D. W., \& Young, T. B. (1994). The relation between cigarette smoking and sleep disturbance. Preventive Medicine, 23(3), 328 334.

Wittmann, M., Dinich, J., Merrow, M., \& Roenneberg, T. (2006). Social jetlag: Mis-alignment of biological and social time. Chronobiology International, 23, 497-509.

Wittmann, M., Paulus, M., \& Roenneberg, T. (2010). Decreased psychological well-being in late 'chronotypes' is mediated by smoking and alcohol consumption. Substance Use \& Misuse, 45(1-2), 15-30.

Wulff, K., Gatti, S., Wettstein, J. G., \& Foster, R. G. (2010). Sleep and circadian rhythm disruption in psychiatric and neurodegenerative disease. Nature Reviews Neuroscience, 11(8), 589-599.

Publisher's Note Springer Nature remains neutral with regard to jurisdictional claims in published maps and institutional affiliations. 